\title{
Evolution and Ecological Adaptations of Microornamentation in Australian Geckos (Gekkota, Squamata)
}

\author{
Jendrian Riedel ${ }^{1,2}$ \\ Thesis submitted for the degree of Doctor of Philosophy, \\ James Cook University
}

\begin{abstract}
Morphological adaptation to different ecological demands is a driver of diversity. Squamate reptiles, including the diverse Australian gecko fauna, have evolved microstructures on their outermost skin surface. Geckos' toepads feature adhesive hair-like setae, while the remaining skin surface features hydrophobic, self-cleaning spinules and small sense organs called cutaneous sensilla. I studied the evolution and ecological adaptation of these microstructures, applying scanning electron microscopy and comparative phylogenetic methods.

I described the diversity of dorsal microstructures of 27 gecko species. Quantifying scale size, spinule length and density, among others, I correlated these traits with habitat use (arboreal, saxicoline or terrestrial) and relative habitat humidity (desert, woodland or rainforest). Terrestrial species had long spinules and more complex sensilla, saxicoline species had larger diameter sensilla, and arboreal species had large granule and small intergranule scales. Desert and woodland species overlapped highly morphologically, differing through smaller-diameter sensilla from rainforest species. These associations suggest that epidermal microstructures evolved in response to environmental variables.

Hydrophobicity is common in nature, but its evolutionary drivers are unclear. I predicted that high hydrophobicity should coevolve with terrestrial microhabitat use, due to higher prevalence of dirt and debris. I reconstructed the evolution of hydrophobicity in 24 gecko species in relation to habitat use, and determined the morphological traits associated with hydrophobicity. Terrestrial species were, indeed, more hydrophobic than arboreal ones. Longer spinules and smaller scales were correlated with high hydrophobicity. Thus, hydrophobicity has co-evolved with terrestrial microhabitat use via selection for long spinules and small scales, likely to keep the skin clean and prevent fouling.

Gecko toepad diversity can be categorised into terminal versus basal toepads. The evolution of toepads is poorly understood because intermediate morphological configurations between pad-less and pad-bearing forms are rare, particularly for terminal toepads. I assessed the subdigital scale morphology of phylogenetically distinct lineages of the mostly terrestrial Bynoe's gecko species complex (Heteronotia binoei): it is described as pad-less, but two distantly related saxicoline lineages have enlarged terminal scales. I reconstructed the ancestral scale size of nine lineages and compared the subdigital microstructures of the saxicoline lineages to their respective sister lineages. The saxicoline lineages independently evolved enlarged terminal scales, and their setae were longer, and branched more often. Thus, the saxicoline lineages evolved more complex adhesive structures in parallel, and their morphology represents a candidate for an intermediate state in terminal toepad evolution.

Broad-scale evolutionary questions require a thorough ecological knowledge of the species analysed, but quantitative ecological data are often scarce, particularly for Australian geckos. Surveying gecko
\end{abstract}

This work is licensed under a Creative Commons Attribution-NonCommercial-NoDerivatives 4.0 International Licence. Individual articles may be copied or downloaded for private, scholarly and not-for-profit use. Quotations may be extracted provided that the author and The Royal Society of Queensland are acknowledged. Queries regarding republication of papers, or parts of papers such as figures and photographs, should be addressed to the Secretary of The Royal Society of Queensland (rsocqld@gmail.com). 
communities from 10 sites, spanning deserts, woodlands and rainforests across Queensland, Australia, I quantified ecological niche and habitat use of 34 species, 9 arboreal, 4 saxicoline, 13 terrestrial and 8 generalist. I quantified perch height and diameter for climbing species and compared them to Anolis 'ecomorph' categories. Species richness was lowest in rainforests and highest in woodlands. Most species used a perch space comparable to the 'trunk-ground' ecomorph, but the genus Strophurus preferred shrubs or twigs of small trees, thus occupying the perch space similar to 'grass-bush' anoles.

${ }^{1}$ College of Science and Engineering, James Cook University, Townsville, QLD 4810, Australia

2 Present Address: Evolutionary Biology, Bielefeld University, 33615 Bielefeld, Germany (Jendrian.riedel@my.jcu. edu.au, jendrian.riedel@uni-bielefeld.de)

Riedel, J. (2021). Evolution and ecological adaptations of microornamentation in Australian geckos (Gekkota, Squamata) [Abstract]. Proceedings of The Royal Society of Queensland, 129, x-xx. https://doi.org/10.53060/ prsq.2021.a2 